\title{
Genetic and phylogenetic analysis of Chinese sacbrood virus isolates from infected Apis mellifera
}

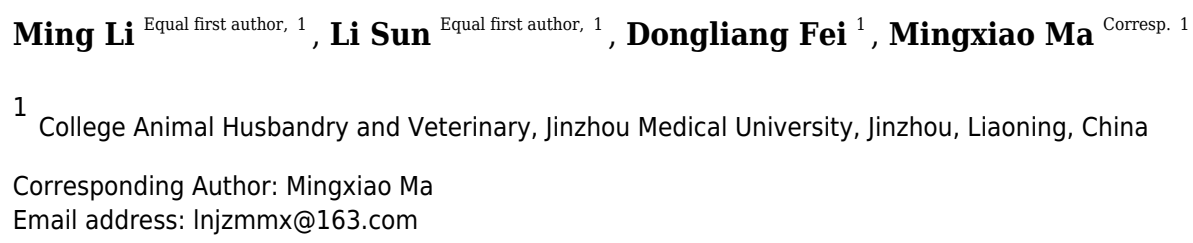

[p] [b] Background.

[b] Sacbrood virus (SBV) is one of the most

pathogenic honeybee viruses that exhibits host specificity and regional

variations. The SBV strains that infect the Chinese honeybee Apis cerana are called Chinese SBVs

(CSBVs). [p]

\section{[p] Methods.}

In this study, a CSBV strain named AmCSBV-SDLY-2016 (GenBank accession No. MG733283) infecting A. mellifera was identified by electron microscopy, its protein composition was analyzed by SDS polyacrylamide gel electrophoresis and agar gel immunodiffusion assay, and its nucleotide sequence was identified using a series of RT-PCR fragments of AmCSBV-SDLY-2016 generated using SBV/CSBV-specific primers. To investigate phylogenetic relationships of the CSBV isolates, a phylogenetic tree of the complete open reading frames of the CSBV sequences was constructed using MEGA 6.0; then, the similarity and recombination events among the isolated CSBV strains were analyzed using SimPlot and RDP4 software, respectively. [p]

\section{[p] Results. Sequencing}

results revealed the complete 8,794-nucleotide long complete genomic RNA of the strain, with a single large open reading frame $(189-8,717)$ encoding 2,843 amino acids. Comparison of the deduced amino acid sequence with the SBV/CSBV reference sequences deposited in the GenBank database identified helicase, protease, and RdRp domains; the structural genes were located at the $5 \phi$ end, whereas the non-structural genes were found at the $3 \phi$ 
acid (aa) and a single aa deletion at positions 711-729 and 2,128, respectively, as compared with CSBV-GD-2002, and a 16-aa deletion (positions 711-713 and 715-728) as compared with AmSBV-UK-2000. However, AmCSBV-SDLY-2016 was similar to the CSBV-JLCBS-2014 strain, which infects A. cerana. AmCSBV-SDLY-2016 ORF shared 92.4 - $97.1 \%$ identity with the genomes of other CSBV strains ( $94.5-97.7 \%$ identity for deduced amino acids). AmCSBV-SDLY-2016 was least similar (89.5 - 90.4\% identity) to other SBVs but showed maximum similarity with the previously reported CSBV-FZ-2014 strain. The phylogenetic tree constructed from AmCSBV-SDLY-2016 and 43 previously reported SBV/CSBV sequences indicated that SBV/CSBV strains clustered according to the host species and country of origin; AmCSBV-SDLY-2016 clustered with other previously reported Chinese and Asian strains (AC genotype SBV, as these strains originated from $A$. cerana) but was separate from the SBV genomes originating from Europe (AM genotype SBV, originating from [i]A. mellifera[i]). A SimPlot graph of SBV genomes confirmed the high variability, especially between the AC genotype SBV and AM genotype SBV. This genomic diversity may reflect the adaptation of SBV to specific hosts, ability of CSBV to cross the species barrier, and the spatial distances that separate CSBVs from other SBVs. [p] 
1 Title page

2 Ms. Ref. No.:

3 Title: Genetic and phylogenetic analysis of Chinese sacbrood virus isolates from Apis mellifera

4 Keywords: Chinese sacbrood virus;A.mellifera;A.cerana;open reading frame;phylogenetic 5 analysis;genomic diversity

6 Authors: Ming $\mathrm{Li}^{\#}$, Li Sun ${ }^{\#}$,Dongliang Fei, Mingxiao $\mathrm{Ma}^{*}$

7 Corresponding Author: Dr. Mingxiao Ma. E-mail: lnjzmmx@163.com

$8 \quad \#$ Co-author: Li Sun

9 Author's Institution:College Animal Husbandryand Veterinary, Jinzhou Medical University,

10 Jinzhou, China

11 First Author: Ming Li

12 Full address: No. 40, section 3 Songpo Road Jinzhou Liaoning Province 121001, China

$13 \quad$ Tel.: +8618904965696

14 Fax:+86 04164673528 
Genetic and phylogenetic analysis of Chinese sacbrood virus isolates from

17 Apis mellifera

\section{Ming Li ${ }^{\#}$ Li Sun ${ }^{\#}$,DongliangFei, Mingxiao Ma*.}

College Animal Husbandryand Veterinary, Jinzhou Medical University, Jinzhou, China

*These authors contributed equally to this study

lnjzmmx@163.com(MMX)

Background. Sacbrood virus (SBV) is one of the most pathogenic honeybee viruses that exhibits host specificity and regional variations. The SBV strains that infect the Chinese honeybee Apis cerana are called Chinese SBVs (CSBVs).

Methods. In this study, a CSBV strain named AmCSBV-SDLY-2016 (GenBank accession No. MG733283) infecting A. mellifera was identified by electron microscopy, its protein composition was analyzed by SDS polyacrylamide gel electrophoresis and agar gel immunodiffusion assay, and its nucleotide sequence was identified using a series of RT-PCR fragments of AmCSBV-SDLY-2016 generated using SBV/CSBV-specific primers. To investigate phylogenetic relationships of the CSBV isolates, a phylogenetic tree of the complete open reading frames of the CSBV sequences was constructed using MEGA 6.0; then, the similarity and recombination events among the isolated CSBV strains were analyzed using SimPlot and RDP4 software, respectively.

Results. Sequencing results revealed the complete 8,794-nucleotide long complete genomic RNA of the strain, with a single large open reading frame $(189-8,717)$ encoding 2,843 amino acids. Comparison of the deduced amino acid sequence with 
the SBV/CSBV reference sequences deposited in the GenBank database identified helicase, protease, and $\mathrm{RdRp}$ domains; the structural genes were located at the $5^{\prime}$ end, whereas the non-structural genes were found at the $3^{\prime}$ end. Multiple sequence alignment showed that AmCSBV-SDLY-2016 had a 17-amino acid (aa) and a single aa deletion at positions 711-729 and 2,128, respectively, as compared with CSBV-GD-2002, and a 16-aa deletion (positions 711-713 and 715-728) as compared with AmSBV-UK-2000. However, AmCSBV-SDLY-2016 was similar to the CSBV-JLCBS-2014 strain, which infects A. cerana. AmCSBV-SDLY-2016 ORF shared 92.4-97.1\% identity with the genomes of other CSBV strains (94.5-97.7\% identity for deduced amino acids). AmCSBV-SDLY-2016 was least similar (89.5-90.4\% identity) to other SBVs but showed maximum similarity with the previously reported CSBV-FZ-2014 strain. The phylogenetic tree constructed from AmCSBV-SDLY-2016 and 43 previously reported SBV/CSBV sequences indicated that SBV/CSBV strains clustered according to the host species and country of origin; AmCSBV-SDLY-2016 clustered with other previously reported Chinese and Asian strains (AC genotype SBV, as these strains originated from $A$. cerana) but was separate from the SBV genomes originating from Europe (AM genotype SBV, originating from $A$. mellifera). A SimPlot graph of SBV genomes confirmed the high variability, especially between the AC genotype SBV and AM genotype SBV. This genomic diversity may reflect the adaptation of SBV to specific hosts, ability of CSBV to cross the species barrier, and the spatial 
60

61

62

63

64

65

distances that separate CSBVs from other SBVs.

\section{Introduction}

Sacbrood virus (SBV) is one of the most pathogenic honeybee viruses that infects larvae and causes larval death. SBV is also known to infect adult bees and reduce the honey production capacity. At present, SBV has been globally found (Allen et al., 1996; Berenyi et al., 2006; Ellis et al., 2005). SBV is a single positive-stranded picornavirus in the genus Iflavirus that encodes one large open reading frame $(\mathrm{ORF})$, with structural genes located at the $5^{\prime}$ end and nonstructural genes at the $3^{\prime}$ end (Chen et al., 2006). The complete genomic sequence of AmSBV-UK-2000 (in this study, we refer to the previously reported SBV/CSBV strains as "SBV/CSBV + isolated geographic location + year") was first determined by Ghosh et al. (Ghosh et al., 1999).

The SBV strains that infect the Asian honeybee Apis cerana are called CSBVs. There is no distinct difference between the nonstructural proteins of SBV and CSBV; however, the structural protein VP1 has the highest variation in amino acid sequence among the proteins from different SBV strains (Cheng et al., 2011). SBV has been divided into two major groups - AC genotype SBV originating from A. cerana and AM genotype SBV from A. mellifera-based on the SBV VP1 sequence (Mingxiao Ma et al., 2013). The AC genotype may be divided further into subgroups. The differences between the AC and AM genotypes may result 
81 from the adaptation of the virus to different hosts and the existence of different

82 subgroups of the AC genotype based on regional variations (Choe et al., 2012;

83 Grabensteiner et al., 2001; Mingxiao Ma et al., 2013). The AC genotype SBV

84 strains were mainly isolated from Asian countries, and their hosts include $A$.

85 cerana (Dali et al., 2012; Guanghua et al., 1979; Kondreddy et al., 2017; Mingxiao

Ma et al., 2011a; Nguyen et al., 2013; Reddy et al., 2016; Se E. et al., 2012; Xia et

al., 2015; Ying et al., 2016; Yu et al., 2013; Zhang et al., 2001). However, the AM genotype SBV persists in the bee colony and may cause infection of $A$. mellifera (Allen et al., 1996; Berenyi et al., 2006; Ellis et al., 2005; Ian et al., 2016;

Khaliunaa et al., 2016; Suresh et al., 2016). The AC genotype isolated from China, infecting the Chinese honey bee $A$. cerana was named as CSBV (Dali et al., 2012; Guanghua al., 1979; Hanrong et al., 2000; Mingxiao Ma et al., 2011a, 2013; Ying et al., 2016; Zhang et al., 2001). CSBV and SBV are similar in structure, physiology, and biochemistry, but have different antigenicity and exhibit no crossinfection (Guanhuang Yang et al., 1979, Jianxun Feng et al., 1999).

Among the $\mathrm{AC}$ genotype SBV strains, the first genome sequence was obtained for CSBV-GD-2002 (Zhang et al., 2001), and subsequently other CSBV genomes, including CSBV-FZ-2014, CSBV-JLCBS-2014, CSBV-LNQY-2008, CSBV-SXYL-2015, and CSBV-BJ-2012, were sequenced. CSBV is similar to AmSBV-UK-2000 in terms of its physiological and biochemical features; however, the viruses differ in their antigenicity and do not show cross-infection (Cheng et al., 
102 2011; Choe et al., 2012; Guanghua et al., 1979; Mingxiao Ma et al., 2013). 103 Sequence analysis indicated that CSBV was different but highly homologous to AmSBV-UK-2000. The genetic characterization and phylogenetic relationship of SBV-infected honeybees collected from different hosts and various geographic 106 regions have recently attracted attention. Previous studies have focused on the 107 alignment, basic structure, and composition of SBV/CSBV genomes, and host108 specificity and geographic differences of SBV/CSBV (Choe et al., 2012;

109 Grabensteiner et al., 2001; Kondreddy et al., 2017; Mingxiao Ma et al., 2011a, 110 2013; Nguyen et al., 2013; Reddy et al., 2016; Se E et al., 2012; Xia et al., 2015;

111 Ying et al., 2016; Yu et al., 2013; Zhang et al., 2001); however, it is unclear 112 whether there is recombination between SBV and CSBV and if CSBV breaks 113 through the species barrier, develops cross-infection, and causes disease (kills 114 larvae) in A. mellifera. Using artificial infection experiments, Hong-Ri Gong et al. 115 demonstrated that CSBV is able to infect $A$. mellifera, but did not observe obvious 116 signs of the disease, which indicated low pathogenicity (Hong-Ri Gong et al., 117 2016).

118 In this study, we characterized a CSBV strain in A. mellifera from Shandong, 119 China (henceforth referred to as AmCSBV-SDLY-2016 [GenBank accession No. 120 MG733283]), and report its molecular and biological characteristics. Furthermore, 121 we analyzed the virus recombination. 
123 Clinical sample collection and testing

124 In total, 359 samples (each collected sample included five larvae from a single 125 colony) were obtained from China between 2008 and 2017. Among all 359 126 samples, 20 samples were from A. mellifera and 359 samples were from A. cerana. 127 In all larvae, CSBV infection was detected by real-time quantitative RT-PCR 128 (qRT-PCR) (Mingxiao Ma et al., 2013). The field studies did not involve 129 endangered or protected species, and the owner of Linyi bee farm Zhang Youcai 130 gave permission to conduct the study on this site.

131 We randomly selected one positive larva for genetic characterization of VP1 132 from the same colony at the same time. The VP1 gene of CSBV was amplified 133 using VP1-specific primers

(F: $5^{\prime}$

R: $\quad 5^{\prime}-$ GCAAGCTTTTATTGTACGCGCGGTAAATA - 3') and sequenced. Phylogenetic tree construction from VP1 CSBV isolates and reference strains in GenBank (Tables 1 and 2) using the MEGA 5.0 package (Tamura et al., 2007) and the neighbor-joining (NJ) method (Saitou et al., 1987). The phylogenetic tree was computed using the Kimura 2-parameter 141 method (Kimura 1980). A bootstrap value of 1,000 replicates was applied to yield 142 a robust phylogeny. We named the samples after the strain that originated in the 143 same region and showed $100 \%$ homology, then submitted the data to GenBank 
144 (CSBV uniformly renamed as CSBV + first isolated geographic location + year).

145

146

147

148

149

150

151

152

153

154

155

156

157

158

159

160

161

162

163

164

\section{Virus purification}

AmCSBV-SDLY-2016 was obtained from a natural outbreak on Linyi bee farm in Shandong, China. A. mellifera larvae infected by AmCSBV-SDLY-2016 were collected by the owner of Linyi bee farm. Fifty infected $A$. mellifera larvae displaying upward warping of the head and body surface changes, and white or sick and dead larvae in the honeycomb with the capped brood abnormally sealed, were collected, weighed, and completely homogenized in sterile water $(1.5 \times$ by weight) using a mortar and pestle. AmCSBV-SDLY-2016 purification was performed by cesium chloride gradient centrifugation, according to the method reported by Ma et al. (Mingxiao Ma et al., 2011a, b). The supernatant was extracted with an equal volume of 1,1,2-trichlorotrifluoroethane before the aqueous phase was layered over a discontinuous $\mathrm{CsCl}$ gradient $\left(1.5 \mathrm{~g} / \mathrm{cm}^{3}\right.$ and $\left.1.2 \mathrm{~g} / \mathrm{cm}^{3}\right)$ and centrifuged at $270,000 \times g$ for $1 \mathrm{~h}$. The material at the $\mathrm{CsCl}$ interface was harvested and adjusted to a volume of $5 \mathrm{~mL}$ (final density $1.38 \mathrm{~g} / \mathrm{cm}^{3}$ ) with $\mathrm{CsCl}$ solution and centrifuged at $270,000 \times g$ overnight. The supernatant was then successively passed through $0.45-\mu \mathrm{m}$ and $0.22-\mu \mathrm{m}$ cell filters. Healthy larvae treated by the same method were used as the negative control. CSBV was subsequently identified by reverse-transcription polymerase chain reaction (RTPCR) to exclude black queen cell virus (BQCV), acute bee paralysis virus (ABPV), chronic bee paralysis virus (CBPV), deformed wing virus (DWV), Kashmir bee 
165 virus (KBV), and Israeli acute paralysis virus (IAPV), following the method 166 reported by $\mathrm{Yu}$ et al. (Yu et al., 2013). CSBV virus samples free of other viruses 167 were stored at $-80^{\circ} \mathrm{C}$ until further use.

168 Electron microscopy and sodium dodecyl sulfate polyacrylamide gel

169

170

171

172

173

174

175

176

177

178

179

180

181

182

183

184

185

\section{electrophoresis (SDS-PAGE) for virus identification}

As previously described (Mingxiao Ma et al., 2011a, b), $100 \mu \mathrm{L}$ of the purified viral suspension was directly pelleted onto carbon-coated Formvar copper grids by ultracentrifugation $(15 \mathrm{~min}$ at $82,000 \times g)$ using a Beckman Airfuge. The grids were negatively stained with $2 \%$ sodium phosphotungstate at $\mathrm{pH} 6.8$ for $90 \mathrm{~s}$ and observed using a Philips CM10 transmission electron microscope.

Structural proteins were separated by SDS-PAGE with 5\% stacking and 12\% separating gels using standard protocols.

\section{Agar gel immunodiffusion (AGID) assay}

Briefly, $1 \mathrm{~g}$ of agarose and $8 \mathrm{~g}$ of sodium chloride $(\mathrm{NaCl})$ were added to 100 mL phosphate buffer (0.01 M, pH 7.2), shaken well, and microwaved for 2 min to prepare an agar solution. The solution was slightly cooled, poured into Petri dishes (90 $\mathrm{mm}$ in diameter; $20-22 \mathrm{~mL}$ of agar per plate), and allowed to solidify. Seven wells were made in the agar plates to identify AmCSBV-SDLY-2016. The central hole was loaded with antisera against the CSBV-JLCBS-2014 strain; the surrounding wells 1 and 2 were loaded with purified AmCSBV-SDLY-2016; wells 3 and 4 were loaded with a known positive control (purified CSBV-JLCBS-2014); 
186

187 The plates were then placed in a closed, moist container and incubated at $37^{\circ} \mathrm{C}$ in

188 a humidified chamber for 24 or $48 \mathrm{~h}$. Precipitation was visible after $24 \mathrm{~h}$ but 189

190

191

192

193

194

195

196

197

198

199

200

201

202

203

204

205

206

and wells 5 and 6 were loaded with treated healthy larvae as the negative control. became more distinct after $48 \mathrm{~h}$ of incubation.

\section{Analysis of pathogenicity}

As described previously (Mingxiao Ma et al., 2013), PCR probes were used to measure the copy numbers of AmCSBV-SDLY-2016. The purified AmCSBVSDLY-2016 was 10-fold serially diluted.

As described previously (Ying Hu et al., 2016), two-day-old $A$. cerana larvae from a single mated queen honeybee in a healthy apiary in Jinzhou, Liaoning Province, were orally inoculated with CSBV. In total, 120 larvae were selected and randomly distributed into six groups $(\mathrm{n}=20$ larvae per group). Each group was inoculated with AmCSBV-SDLY-2016 $\left(1.25 \times 10^{4}, 1.25 \times 10^{5}, 1.25 \times 10^{6}, 1.25 \times\right.$ $10^{7}$, and $1.25 \times 10^{8}$ copies/larva in groups $1-6$, respectively). The assay was performed in triplicate. The virus inoculation assay was performed twice after the end of the first experiment.

Each larva was fed $20 \mu \mathrm{L}$ of a virus suspension mixed with an equal amount of basic larval diet (BLD, Liu et al., 2010) consisting of 50\% royal jelly, 37\% sterile water, $6 \%$ glucose, $6 \%$ fructose, and $1 \%$ yeast extract, at $95 \%$ relative humidity and $34^{\circ} \mathrm{C}$. The virus-free control was fed $20 \mu \mathrm{L}$ of sterile water with an equal amount of BLD. BLD was used subsequently for daily feeding. The clinical signs 
207 in each group of larvae were examined and recorded every day until larvae death.

208 All larvae that were orally inoculated in this study were analyzed by RT-PCR for

209

210

211

212

213

214

215

216

217

218

219

220

221

222

223

224

225

226

227

BQCV (Grabensteiner et al., 2007), ABPV (Grabensteiner et al., 2007), CBPV

(Tentcheva et al., 2004), DWV (Tentcheva et al., 2004), KBV (Blanchard et al., 2014), IAPV (Miranda et al., 2010), and qRT-PCR for CSBV (Mingxiao Ma et al., 2013).

The statistical analysis of larval mortality was performed between groups and between three replicates of each group using SPSS 22.0.

\section{AmCSBV-SDLY-2016 genome sequencing}

The primers used in this study (Table 3) were designed based on the nucleotide sequences of CSBV-JLCBS-2014, AmSBV-UK-2000, and CSBV-GD-2002. The full-length AmCSBV-SDLY-2016 genome was determined by Sanger sequencing of the RT-PCR fragments and $3^{\prime}$ rapid amplification of cDNA ends (RACE) (Clontech-Takara, Mountain View, CA), according to the method described by Ma et al. (Mingxiao Ma et al., 2011a). The PCR amplification product was cloned into the pMD-18-T vector (Takara Biotechnology Co. Ltd., Dalian, China). The plasmids were then used to transform Escherichia coli DH5 $\alpha$ cells (Takara Biotechnology Co. Ltd). For each RT-PCR fragment, five clones were randomly chosen and sequenced by Sangon Biotech Co. Ltd. If all five clones were identical, the sequence was considered correct. The correct nucleotide sequences from all the fragments were assembled to build a continuous complete 
228

229

230

231

232

233

234

235

236

237

238

239

240

241

242

243

244

245 246 strains were divided into nine groups as follows: A (CSBV-FZ-2014), B 247 (AmCSBV-SDLY-2016), C (CSBV-SXnor-2012), D (CSBV-SXYL-2015), E 248 (CSBV-BJ-2012), F (CSBV-LNQY-2008), G (CSBV-JLCBS-2014), H (CSBV-

sequence using the DNASTAR software, and the VP1 gene sequence was analyzed as described above.

\section{Phylogenetic tree construction from AmCSBV-SDLY-2016 and SBV/CSBV}

\section{genome sequences}

Nucleotide sequences of the amplified RT-PCR fragments were assembled to generate the entire genome of AmCSBV-SDLY-2016 using the DNASTAR program. Multiple nucleotide and deduced amino acid sequence alignments identified sequence variations between clones covering the same SBV genome regions using ClustalW in the MegAlign program (DNAStar Inc., Madison, WI, USA) and the published SBV/CSBV sequences. A phylogenetic tree was constructed from the nucleotide sequences of the coding regions of 43 previously reported SBV/CSBV strains from various countries and the AmCSBV-SDLY-2016 isolated in this study. The phylogenetic tree constructed using the MEGA 6.0 package (Tamura et al., 2007) and NJ method (Saitou et al., 1987) was computed using the Kimura 2 parameter method (Kimura 1980). The phylogenetic tree was bootstrapped 1000 times and bootstrap values placed on each branch.

\section{Similarity and virus recombination analysis}

45 Based on the results of the phylogenetic analysis, the isolated CSBV/SBV 
249 GD-2002), and I (AmSBV-UK-2000) for similarity analysis and virus 250 recombination analysis. The Recombination Detection Program (RDP) values were 251 computed using the RDP 4.0 software for the preliminary screening of the major 252 parent and minor parent sequences. Similarity plots and BootScan were computed 253 using Simplot software (Lole et al., 1999) with the following parameters: a 254 window of 200 base pairs (bp; step: 20 bp), with gap-stripping and Kimura (2255 parameter) correction, using AmCSBV-SDLY-2016, CSBV-JLCBS-2014, CSBV256 GD-2002, and AmSBV-UK-2000 for Find Sites, and AmCSBV-SDLY-2016 and 257 CSBV-JLCBS-2014 as the query sequences.

RESULTS

259

Characteristics of the VP1 gene

260

Of the 359 samples tested, 326 were found to be positive by strand-specific 261 qPCR, 18 of which originated from A. cerana, whose infection rate was $90.0 \%$ $262(18 / 20)$, and the $95 \%$ confidence intervals for infection rate were $69-99 \%$, with 308 263 positive samples originating from $A$. mellifera whose infection rate was $90.9 \%$ $264(308 / 339)$, and the $95 \%$ confidence intervals for the infection rate were 85.90 $26595.81 \%$. Positive samples originating in the same region that showed $100 \%$ 266 homology were defined as a strain. Thirty-one CSBV strains were isolated 267 according to the aforementioned virus-naming scheme.

268 The phylogenetic tree of VP1 revealed two clusters (Fig. 1). One was related to 269 SBV strains that originated from A. cerana (AC genotype), the other was related to 
the SBV strains that originated from A. mellifera (AM genotype). The phylogenetic

271 tree also showed that AmCSBV-SDLY-2016 originating from A. mellifera

272 belonged to the clade containing the CSBV and other Asian strains. To simplify

273 the naming of the strain, we used AmSBV and AmCSBV to represent the

$274 \mathrm{SBVs} / \mathrm{CSBV}$ strains isolated from $A$. mellifera; all the others represent the

275 SBVs/CSBV strains that were isolated from A. cerana. The corresponding host and 276 gene numbers are listed in Tables 1 and 2.

277

278

\section{AmCSBV-SDLY-2016 identified by electron microscopy and SDS-PAGE}

Electron microscopy showed large amounts of typical CSBV particles in the preparations from virus-infected larvae; CSBV particles were icosahedrons and had an approximate diameter of $26 \mathrm{~nm}$ (Fig. 2). No virus particles were observed in the control preparations from healthy larvae. The four main structural proteins of CSBV were separated by SDS-PAGE (Fig. 3). The molecular weights of the four proteins were about 44.2, 37.8, 31.5, and $30.5 \mathrm{kDa}$, respectively (Jianxu et al., 1998; Mingxiao Ma et al., 2011c).

\section{AmCSBV-SDLY-2016 identified by AGID assay}

AGID assay revealed the distinct CSBV-specific lines of precipitin observed between the wells containing AmCSBV-SDLY-2016 and the antisera against

290 lines were observed in the negative control wells. 
291

292

293

294

295

296

297

298

299

300

301

302

303

304

305

306

307

308

309

310

311

Pathogenicity

After oral inoculation with AmCSBV-SDLY-2016 (groups 1-5), all larvae were analyzed by RT-PCR and qRT-PCR. All of the other honeybee viruses were undetectable, whereas CSBVs were detectable. All the larvae in the virus-free control (group 6) lacked common honeybee viruses.

Three repeated experiments showed that upon inoculation with $1.25 \times 10^{4}$ copies, $1.25 \times 10^{5}$ copies, or $1.25 \times 10^{6}$ copies of AmCSBV-SDLY-2016, larvae mortality rates were $35-45 \%, 65-75 \%$, and $80-90 \%$, whose $95 \%$ confidence intervals for the proportion dead pupae were $19-64 \%, 46-88 \%$, and $62-97 \%$, respectively (Table 4). In contrast, mortality of the larvae was $100 \%$ in the two groups, where each larva was sequentially inoculated with $1.25 \times 10^{7}$ and $1.25 \times$ $10^{8}$ copies of AmCSBV-SDLY-2016, whose 95\% confidence intervals for the proportion dead pupae were $83-100 \%$. In the virus-free control groups, the mortality rates of the larvae were $20-25 \%$, with $95 \%$ confidence intervals for the proportion dead pupae of $6-44 \%$.

Statistical analysis showed that there were no significant differences between the larval mortality from three repeated experiments in each group, but there were highly significant differences in larval mortality between each group, except that there was no difference in the number of larval mortality between the two groups, where each larva was sequentially inoculated with $1.25 \times 10^{7}$ and $1.25 \times 10^{8}$ copies of AmCSBV-SDLY-2016. 
312 Nucleotide sequence

313 The nucleotide sequence of the AmCSBV-SDLY-2016 genome was 8,794-bp

314 long, and the percentages of $\mathrm{A}, \mathrm{U}, \mathrm{G}$, and $\mathrm{C}$ were $29.95 \%, 29.22 \%, 24.30 \%$, and $31516.52 \%$, respectively, similar to those reported for other CSBV/SBV strains 316 encoding one large ORF. Two AUG codons were located at positions 189 and 429 317 of the AmCSBV-SDLY-2016 genome; however, AUG 189 is likely the translation 318 initiation site, as unlike AUG 429, it was observed in a sequence (AUUAUGG) 319 identical to that of many invertebrate initiation codons (ANNAUGG). The size of 320 the CSBV 3' untranslated region (UTR) (77 nucleotides) was similar to that from 321 other picornaviruses (40-126 nucleotides). The 5' UTR was generated from 322 AmCSBV-SDLY-2016 by RT-PCR with a primer designed using the CSBV323 JLCBS-2014 sequence, indicating that the CSBV 5' UTR was similar to that of 324 CSBV-JLCBS-2014 (188 nucleotides). Multiple sequence comparisons revealed a 325 sequence homology of $92.4 \%$ to $97.1 \%$ among all CSBV isolates and a similarity 326 of $94.5 \%$ to $97.7 \%$ in the deduced amino acid sequences. AmCSBV-SDLY-2016 327 was least similar (89.5\% to $90.4 \%$ identity) to other SBVs but showed maximum 328 similarity with CSBV-FZ-2014 (97.1\% and 97.7\% homology of sequence and 329 deduced amino acid sequences, respectively). The results of VP1 sequencing were 330 completely consistent, so we could rule out the possibility that several CSBV 331 isolates could co-exist in the samples originating from $A$. mellifera.

\section{Protein sequence}


333 The deduced amino acid sequences of AmCSBV-SDLY-2016 genomes and 334 previously reported SBV/CSBV strains were aligned and compared. Results 335 revealed that the structural and non-structural proteins were located at the $5^{\prime}$ and $3^{\prime}$ 336 ends, respectively. Multiple sequence alignment showed that AmCSBV-SDLY3372016 had 17 and 1 amino acid deletions (positions 711-729, which are in the region 338 of structural protein VP1, and 2,128, which is in the region of nonstructural protein) 339 as compared with CSBV-GD-2002, and 3 and 13 amino acid deletions (positions 340 711-713 and 715-728, which are in the region of structural protein, respectively) as 341 compared to AmSBV-UK-2000(Fig.5). However, AmCSBV-SDLY-2016 was 342 similar to the CSBV-JLCBS-2014 strain that infects $A$. cerana.

343 The amino acid sequence at the C-terminal region of the AmCSBV-SDLY-2016 344 polyprotein was similar to that for helicase, protease, and RNA-dependent RNA 345 polymerase $(\mathrm{RdRp})$ domains of the previously reported SBV/CSBV strains. The 346 highly conserved amino acid motifs GPAGIGKS, QPVVVYDD, and 347 KKIRGNPLIVILLCNH, corresponding to the helicase domains A, B, and C 348 (Supplementary 1), respectively, were located between the amino acid positions 349 1,353 and 1,473; however, the $\mathrm{C}$ domain containing KKIRGNPLIVILLCNH 350 appeared to be the most conserved, unlike mammalian picornaviruses. The 351 conserved cysteine protease motif ${ }^{2249} \mathrm{GXCG}^{2252}$ (GACG) and the putative 352 substrate-binding residue ${ }^{2266} \mathrm{GxHxxG}^{2271}$ (GMHFAG) were detected in the $3 \mathrm{C}$ 353 protease domains spanning amino acids 2,141 to 2,272 (Supplementary 2). In 
354 addition, eight conserved domains identified in RdRp were also detected between 355 amino acid positions 2,444 and 2,813 in AmCSBV-SDLY-2016 (Supplementary 3). 356

\section{Phylogenetics}

358 To assess the genetic relationship among the SBV/CSBV strains, a phylogenetic 359 tree based on the nucleotide sequence of the SBV/CSBV-coding region was 360 constructed by the NJ method for AmCSBV-SDLY-2016 and all SBV/CSBV 361 reference strains in GenBank. The tree was similar to the phylogenetic tree of VP1, 362 which revealed two clusters, one related to SBV strains that originated from $A$. 363 cerana (AC genotype), and the other related to the SBV strains that originated 364 from A. mellifera (AM genotype) (Fig. 6). The AM cluster was distinctly separate 365 from the AC lineages, as supported by a clade credibility value of 100.0 . The AC 366 genotype was further subdivided into several subtypes according to their countries 367 of origin and host species.

368 The phylogenetic tree also showed that AmCSBV-SDLY-2016 belonged to the 369 clade containing the CSBV and other Asian strains. These results indicate that the AmCSBV-SDLY-2016 strain showed close genetic relationships with CSBV 371 strains, specifically CSBV-FZ-2014.

\section{Similarity profile}

373 To identify differences in the full-length sequences and various genomic 374 regions from CSBV strains AmSBV-UK-2000 and AmCSBV-SDLYA-2016, the 
375 complete coding regions were plotted using SimPlot, with AmCSBV-SDLY-2016 376 as the query sequence (Fig. 7). AmCSBV-SDLY-2016 from A. mellifera was more 377 similar to the CSBV strains isolated than AmSBV-UK-2000. The CSBV strains 378 isolated formed a relatively independent separation group; there was an obvious 379 deviation compared to AmSBV-UK-2000, with maximum deviation (19.3\%) 380 occurring at 2,181 and 2,221 bp. A high degree of consistency was observed 381 between the genomes of AmCSBV-SDLY-2016 and isolated CSBV strains, with 382 more than $85 \%$ similarity between the complete coding regions. Among all the 383 isolated CSBV strains, only recombinant CSBV-JLCBS-2014 was detected by 384 385 386 387 388 389 390

Discussion

392 CSBV was first described in Guangdong in 1972; it later disseminated to the rest 393 of China and Southeast Asian countries, causing a lethal disease in individual bees 394 or the collapse of entire colonies, and was termed as "bee cancer" by beekeepers 395 (Mingxiao Ma et al., 2010). More than 200,000 colonies (more than half of the 
396 total number of local colonies) were destroyed in Guangxi and Guangdong 397 provinces from 1972 to 1994 (Hanrong et al., 2000). It re-emerged and caused the 398 collapse of entire colonies in Liaoning China in 2008 (Dali et al., 2012; Mingxiao 399 Ma et al., 2010). Although CSBV and SBV are similar in structure, physiology, 400 and biochemistry, these viruses differ in antigenicity and cross-infection 401 (Guanghua et al., 1979). SBV strains usually show host specificity in the structural 402 polyprotein regions of their genomes (Cheng et al., 2011; Choe et al., 2012; 403 Mingxiao Ma et al., 2013). CSBV, which has been divided into different subtypes 404 according to various geographic regions and host specificity (Mingxiao Ma et al., 405 2013), contains three structural proteins, namely, VP1, VP2, VP3, and an unknown 406 protein with a molecular weight of $44.2 \mathrm{kDa}$ (Jianxu et al., 1998; Mingxiao Ma et 407 al., 2011c). SBV contains three structural proteins (VP1, VP2, and VP3). The 408 proteins VP1, VP2, and VP3, as well as the unknown 44.2 kDa protein of CSBV, 409 were detected by SDS-PAGE on CSBV strains (termed as AmCSBV-SDLY-2016) 410 that infect $A$. mellifera, and distinct CSBV-specific lines of precipitation were 411 observed between AmCSBV-SDLY-2016 and the antisera against CSBV-JLCBS4122014 in the AGID assay. Thus, AmCSBV-SDLY-2016 belongs to CSBV. As 413 expected, the phylogenetic and similarity analyses showed that the AmCSBV414 SDLY-2016 genome and the specific VP1 gene isolated from $A$. mellifera were 415 highly similar to the genomes and specific VP1 gene of CSBV strains derived from 416 A. cerana. Similar to AmCSBV-SDLY-2016, two Vietnamese SBVs strains 
417 (AmSBV-Vt-4-2014 and AmSBV-Viet6-2014) and two Korean strains (AmSBV418 Kor19-2012 and AmSBV-Kor2-2016) isolated from A. mellifera also belong to the 419 AC type (Fig. 6), consistent with previous reports (Kondreddy et al., 2017; Se E et 420 al., 2012). Although no reports are available to determine if this strain caused $A$. mellifera larval deaths, the results of the present study show that the long-term existence and widespread prevalence of the AC type SBV in nature may lead to 423 exchange of viruses among host populations. Furthermore, phylogenetic and 424 similarity analyses showed that the virus strains from the same continent or country have higher levels of similarity and genetic clustering.

426

The phylogenetic tree based on the nucleotide sequences of the SBV/CSBV427 coding region revealed the genetic relationship between different virus isolates 428 obtained from various geographic areas and hosts. Two distinct branches were 429 formed according to bee species, and a higher level of similarity was observed 430 between viral genomes from the same country, continent, or neighboring countries.

431 Furthermore, CSBV genome sequences were closely related to those from Vietnam, 432 Korea, and India, all of which formed a neighboring subcluster. The phylogenetic 433 trees based on the SBV/CSBV-coding region and VP1 nucleotide sequences were 434 identical (Mingxiao Ma et al., 2013), indicating that SBV/CSBV strains may be 435 divided into different groups, and that VP1 may replace the complete genomic 436 sequence of SBVs and CSBVs and be used as a specific target for studying 437 genotyping, genetic evolution, and molecular epidemiology of SBV/CSBV strains 
438 in the future.

439 Direct sequencing of virus genome amplicons and comparison with known 440 nucleotide sequences or deduced protein sequences of virus isolates obtained from 441 various geographic areas and hosts may reveal the genome organization and 442 molecular basis of pathogenicity of the newly isolated strains. Analysis of the 443 deduced amino acid sequences showed that AmCSBV-SDLY-2016, like CSBV, 444 comprised conserved motifs within the helicase, protease, and RdRp domains. 445 Furthermore, AmCSBV-SDLY-2016 from CSBVs was genetically similar to the 446 previously reported strains CSBV-FZ-2014 and CSBV-JLCBS-2014. We observed 447 nucleotide transitions and insertions in the entire genome sequences, thereby 448 reflecting the divergence of these genomes. In comparison to the CSBV strains that 449 were isolated prior to 2014 (CSBV-GD-2002, CSBV-LNQY-2008, CSBV-FZ450 2014, CSBV-SXnor-2012, and CSBV-BJ-2012), AmCSBV-SDLY-2016, CSBV451 JLCBS-2014, CSBV-SXYL-2015, and AmSBV-UK-2000 strains carried a deletion 452 mutation at the amino acid residue 2,128, and AmCSBV-SDLY-2016 and CSBVSXYL-2015 differed at positions 711-729. We speculated that if AmCSBV-SDLY4542016 could cross the species barrier and cause death in $A$. mellifera larvae, then 455 CSBV-JLCBS-2014 could probably cross the species barrier and kill $A$. mellifera 456 larvae because of the similarity at positions 711-729 and 2,128. In addition, we 457 demonstrated that CSBV-JLCBS-2014 was a recombinant virus, and the sequence 458 of its parent strain was derived from AmCSBV-SDLY-2016, CSBV-FZ-2014, and 
459 CSBV-LNQY-2008 as determined by RDP and BootScan. However, AmCSBV460 SDLY-2016 was isolated after CSBV-JLCBS-2014, and the recombination signals 461 obtained from CSBV-FZ-2014, CSBV-JLCBS-2014, and CSBV-GD-2002 were 462 detected by BootScan (Fig. 7). Hence, we speculate that AmCSBV-SDLY-2016 463 may be a recombinant virus strain and its parent strain was derived from CSBV464 FZ-2014, CSBV-JLCBS-2014, and CSBV-GD-2002.

465 CONCLUSIONS

466

In summary, the strain AmCSBV-SDLY-2016 of CSBV is genetically similar 467 to the previously reported strains of CSBV that infect A. cerana; however, 468 AmCSBV-SDLY-2016 may cross the species barrier, and infect and cause death in 469 A. mellifera larvae. This observation shows that under natural selection and 470 immune pressure, CSBV/SBV strains may be exchanged between hosts, indicative 471 of the independent evolution of these viruses. Future studies will evaluate genetic 472 variations and population structures of CSBV/SBV strains infecting A. mellifera 473 and A. cerana in China and other countries.

\section{$474 \quad$ Funding}

475 This work was supported by grants from the National Science Foundation of China 476 (grant No. 31772760) and Liaoning Natural Science Foundation (grant No. 477 20180550289).

\section{Competing interests}

479 The authors declare no competing interests. 
480

481

482

483

484

485

486

487

488

489

490

491

492

493

494

495

496

497

498

499

500

\section{REFERENCES}

Allen, M. , Ball, B. 1996.The incidence and world distribution of honey bee viruses. Bee World77(3):141-162.

Berenyi, O., Bakonyi, T., Derakhshifar, I., Koglberger, H., Nowotny, N.. 2006. Occurrence of six honeybee viruses in diseased Austrian apiaries. Appl.Environ. Microbiol.72 (4):2414-2420.

Blanchard P, Carletto J, Siede R, Schurr F, Thiéry R, Ribière M. 2014. Identification of Kashmir bee virus in France using a new RT-PCR method which distinguishes closely related viruses. Journal of virological methods 198 , 82-85, doi:10.1016/j.jviromet.2013.12.006.

Chen, Y., Evans, J., Feldlaufer, M.. 2006. Horizontal and vertical transmission of viruses in the honey bee, Apismellifera. J. Invertebr. Pathol.92(3):152-159.

Cheng, J., Zhang, P., Ma, M., Li Ming, Yang Song. 2011.Predication of spatial structure and B cellepitope of VP1 protein of Chinese sacrbrood virus LN-QY strain. Chinese Journalof Biologicals42 (3):280-284.

Choe, S.E., Nguyen, T.T.D., Hyun, B.H., Noh, J.H., Lee, H.S., Lee, C.H., Kang SW. 2012. Genetic and phylogenetic analysis of South Korean sacbrood virusisolates from infected honey bees (Apiscerana). Vet. Microbiol.157(12):32-40.

Dali Zhang. 2012.Investigation and analysis on the occurrence ofChinese Sacbrood virus disease in Liaoning Province. Apiculture of china63:19. 
501 Ellis, J.D., Munn, P.A.. 2005. The worldwide health status of honey bees. 502 BeeWorld86(4):88-101.

503 Ghosh, R.C., Ball, B.V., Willcocks, M.M., Carter, M.J..1999.The 504 nucleotidesequence of sacbrood virus of the honey bee:an insect picorna505 likevirus. J. Gen. Virol.80 (Pt. 6):1541-1549.

506

507

508

509

510

511

512

513

514

515

516

517

518

519

520

521

Grabensteiner, E., Bakonyi, T., Ritter, W., Pechhacker, H. \&Nowotny, N. 2007.Development of a multiplex RT-PCR for the simultaneous detection of three viruses of the honeybee (Apismellifera L.): acute bee paralysis virus, Black queen cell virus and Sacbrood virus. Journal of invertebrate pathology.94, 222225, doi:10.1016/j.jip.2006.11.006.

Grabensteiner, E., W. Ritter, M. J. Carter, S. Davison, H. Pechhacker, J.Kolodziejek,Boecking $\quad$ O, Derakhshifar I, Moosbeckhofer $\quad$ R, Licek E, Nowotny N.. 2001. Sacbrood virus of the honeybee (Apismellifera): rapididentification and phylogenetic analysis using reverse transcriptionPCR.Clin.Diagn.Lab. Immunol.8(1):93-104.

Guanghua Y, Gongshao Z, Zhilan D.1979. Pathogen identification of Chinese sacbroodvirus disease.Chinese beekeeping(5):17-18.

Guanhuang Yang, Gongzhao Zhang, Zhilang Du. 1979.Identification of the pathogen of ChineseSacbrood disease.China apiculture.(5): 17-18.

Hanrong Qin.2000.Prevention and treatment ofChinsessacbrood.Guangxi Animal husbandry and veterinary16(4):28-31. 
522

523

524

525

526

527

528

529

530

531

532

533

534

535

536

537

538

539

540

541

542

Hongri Gong, Xiuxian Chen, Yanping Chen, Hu FL, Zhang JL, Lin ZG, Yu JW, Zheng HQ. 2016. Evidence of ApisceranaSacbrood virus Infection in Apismellifera. Appl Environ Microbiol.82:2256-2262.

Ian CAVIGLI, Katie F. DAUGHENBAUGH, Madison MARTIN,Michael Lerch,Katie Banner,Emma Garcia,Laura M. Brutscher, Michelle L.2016. Pathogen prevalence and abundance in honey bee colonies involved in almond pollination. Apidologie47: 251-266.

JianxuFeng, Zhouying Lu, Qinfen Zhang, Zewei Ma, Jingqiang Zhang, Wenzhong Huang.1998. Studies on purification, Crystallization and structure of Chinese sacbrood virus, J. Chin Electr.Microsc. Soc.17(4):387-388.

Jianxunfeng, Jianxiu Yu, Jingqiang Zhang. 1999. Cloning ofPartial Structure Protein Genefrom Chinese Sacbrood Virus. Journal of Sun Yat-sen University, 38(5):124-126.

KhaliunaaTsevegmid, Peter Neumann, Orlando Yañez. 2016. The Honey Bee Pathosphere of Mongolia: European Viruses in Central Asia. PLoS ONE11(3): e0151164.

Kimura, M. 1980. A simple method for estimating evolutionary rates of basesubstitutions through comparative studies of nucleotide sequences. $J$. Mol.Evol.16(2):111-120.

KondreddyEswar Reddy, Ha Thi Thu, Mi Sun Yo o, MummadireddyRamya, BheemireddyAnjana Reddy, Nguyen Thi Kim Lien, Nguyen Thi Phuong 
Vitro and its Application. Journal of bee.30: 32-34. immunodeficiency virus type 1 genomes from subtype C-infected seroconverters inIndia, with evidence of intersubtype recombination. Journal of Virology73(1):152-160.

Mingxiao Ma, Chen MA, Jian Cheng, ZhenshengXie, Ming Li, Dong-Liang

Fei. 2011c. Prediction and Identification of Structural Proteinsof Chinese

Sacbrood Virus LN Isolate.Chemical Journal of Chinese universities32(8): 1768-1773.

Mingxiao Ma, Jinhua Liu, Yingjin Song, Li Li,Yongfei Li.2013.TaqMan MGB

Probe Fluorescence Real-Time QuantitativePCR for Rapid Detection of Chinese 
564

565

566

567

568

569

570

571

572

573

574

575

576

577

578

579

580

581

582

583

584

Mingxiao Ma, Ming L, Jian C, Song Y, Shude W, Pengfei L. 2011a.Molecular and Biological Characterization of Chinese Sacbrood Virus LN Isolate. Comparative and functionalgenomics(3):409386.

Mingxiao Ma, Yanna Y, Xiaoli X, Lin Z, Yongfei L, Zhidong L. 2013. Genetic characterization of VP1 gene of seven Sacbroodvirus isolated from three provinces in northern Chinaduring the years 2008-2012. Virus Research176(12):78-82.

Mingxiao, Ma, Ming Li, Chunying Yuan, Pengfei Li, Yibo Zhang, Yuhong Su.2010.Development of a RT-PCR methodfor determination of Chinese sacbrood virus. Chinese Journal of Biologicals4:425-427.

Miranda, J. R. D., Cordoni, G., Budge, G.2010. The Acute bee paralysis virusKashmir bee virus-Israeli acute paralysis virus complex.Journal of invertebrate pathology. 103(1): 30-47, doi:10.1016/j.jip.2009.06.014 .

Nguyen NT, Le TH. 2013.Complete Genome Sequence of Sacbrood Virus Strain SBM2, Isolated from the Honeybee Apiscerana in Vietnam. Genome Announc1(1): e00076-12.

Reddy KE, Yoo MS, Kim YH, Kim NH, Ramya M, Jung HN, Le Thi Bich Thao, Hee-Soo Lee, Seung-Won Kang.2016.Homology differences between complete Sacbrood virus genomes from infected Apismellifera and Apisceranahoneybees in Korea.Virus Genes.52(2):281-9.

Saitou, N., Nei, M.. 1987.The neighbor-joining method: a new method for 
reconstructing phylogenetic trees. Mol. Biol. Evol. 4(4):406-425.

586

587

588

589

590

591

592

593

594

595

596

597

598

599

600

601

602

603

604

605

Se E. Choe, Lien T.K. Nguyen, Jin H. Noh, Chang H. Kweon, Kondreddy E. Reddy, Hong B. Koh, Ki Y.Chang, Seung W.Kang. 2012.Analysis of the complete genome sequence of two Korean sacbrood viruses in the Honey bee, Apismellifera. Virology432:155-161.

Suresh D. Desai, Robert W. 2016.Currie.Effects of Wintering Environment and Parasite-Pathogen Interactions on Honey Bee Colony Loss in North Temperate Regions.PLoS ONE11(7):e0159615.

Tamura, K., Dudley, J., Nei, M., Kumar, S. 2007. MEGA4: Molecular Evolutionary Genetics Analysis (MEGA) software version 4.0. Mol. Biol. Evol. 24(8):1596-1599.

Tentcheva D, Gauthier L, Zappulla N, Dainat B, Cousserans F, Colin ME, Bergoin M. 2004.Prevalence and seasonal variations of six bee viruses in Apismellifera L. and Varroa destructor mite populations in France.Applied and environmental microbiology.70:7185-7191, doi:10.1128/AEM.70.12.7185-7191.

Xia X, Zhou B, Wei T. 2015.Complete genome of Chinese sacbrood virus fromApisceranaand analysis of the 3C-like cysteine protease.VirusGenes50(2):277-85.

Ying Hu, Dongliang Fei, Lili Jiang, Dong Wei, Fangbing Li, Qingyun Diao,Mingxiao Ma.2016.A comparison of biological characteristics of three strains of Chinese sacbrood virus in Apiscerana.Scientific Reports.6:37424. 
606 Ying Hu, DongliangFei, Lili Jiang, Dong Wei, Fangbing Li, QingyunDiao, 607 Mingxiao Ma. 2016. A comparison of biologicalcharacteristics of three 608 strainsof Chinese sacbrood virus in Apiscerana. Scientific Reports.6:37424 | 609 DOI: $10.1038 /$ srep37424.

610 Yu H, Liu TX, Wang D.2013. Genomic characterization and phylogenetic analysis 611 of Chinese sacbrood virus isolated from Loess Plateau, China. 612 GenomeAnnounc.1(1).pii:e00076-12.

613 Zhang, J., Feng, J., Liang, Y., Cheng, D., Zhou, Z.H., Zhang, Q. 614 2001.Threedimensional structure of the Chinese sacbrood bee virus. Science in 615 China.44(4):443-448.

616

617

618

619

620

621

622

623

624 
625 Figure captions

626 Fig. 1 Phylogenetic analysis of the VP1 region amino acid sequences obtained

627 from all the CSBV isolates and reference strains in GenBank.

628 The tree reveals two clusters, the AC and AM genotypes.

$629 *$ To simplify the naming of the strain, we used AmSBV and AmCSBV to

630 represent the SBVs/CSBV strains isolated from $A$. mellifera; all the others

631 represent the SBVs/CSBV strains that were isolated from $A$. cerana. The

632 corresponding host and gene numbers are listed in Tables 1 and 2

633 Fig. 2 CSBV particles were observed with electron microscopy.

634 Virus particles of approximately 26-nm diameter were observed in virus 635 preparations from the infected larvae by electron microscopy.

636 Fig. 3 The four proteins of CSBV were detected by SDS-PAGE.

637 The proteins were resolved on $12 \%$ SDS-polyacrylamide gels according to 638 standard protocols. A. representative protein markers.B. virus-free control. C. 639 AmCSBV-SDLY-2016.

640 Fig. 4 Identification of AmCSBV-SDLY by AGID assay.

641 Antisera CSBV(center well), AmCSBV-SDLY-2016 (wells 1 and 2), known 642 positive controlCSBV-JLCBS-2014 (wells 3 and 4), and negative control (wells $643 \quad 5$ and 6$)$.

644 Fig. 5 Protein domain alignments. AmCSBV-SDLY-2016 had 17 and 1 amino 
acid deletions (positions 711-729 and 2,128, respectively). However, AmCSBV-

SDLY-2016 had 3 and 13 amino acid deletions (positions 711-713 and 715-728,

647 respectively) as compared with AmSBV-UK-2000

Fig. 6 Phylogenetic analysis of all nucleotide sequences obtained from various countries, including China, Korea, Vietnam, India, Australia, and the United Kingdom.

651

Numbers on the nodes indicate clade credibility values. Previously reported

652 sequence names are presented in the following format: strains-GenBank accession numbers. The tree reveals two clusters, the AC and AM genotypes.

* The bar represents a genetic distance of 0.01 . CSBV is marked with a red box, 655 the blue square represents that the AC genotype SBVs strains mainly were 656 isolated from $A$. mellifera.

Fig.7 SimPlot graphs comparing AmCSBV-SDLY-2016 with other CSBVs and AmSBV-UK-2000

Each colored line indicates a group of SBV/CSBV strains (for interpreting the color key in this figure legend, please refer to an online version of the article).

Fig. 8 BootScan analysis of the recombinant sequence based on the comparison between the complete coding regions of AmCSBV-SDLY-2016 and other CSBVs and AmSBV-UK-2000.

A. BootScan was conducted with the strain AmCSBV-SDLY-2016 as the query 
666 query sequence. Note: The cross recombination position is labeled by red lines. 667 


\section{Table 1 (on next page)}

Table 1. 31 CSBV strains isolated 
Table 1.31 CSBV strains isolated

\begin{tabular}{|c|c|c|c|c|}
\hline N0. & isolated strain & region & host & GenBank number \\
\hline 1 & CSBV-LNQY-2008 & Qingyuan, Liaoning & A. cerana & HM237361 \\
\hline 2 & CSBV-LNBX-2009 & Benxi, Liaoning & A. cerana & JX854438 \\
\hline 3 & CSBV-LNSZ-2011 & Suizhong, Liaoning & A. cerana & JX854441 \\
\hline 4 & CSBV-JLCC-2011 & Changchun,JiLin & A. cerana & JX854437 \\
\hline 5 & CSBV-LNND-2011 & Kuandian, Liaoning & A. cerana & JX854439 \\
\hline 6 & CSBV-HBQHD-2012 & Qinghuangdao, Hebei & A. cerana & JX854436 \\
\hline 7 & CSBV-LNQY-2012 & Qingyuan, Liaoning & A. cerana & JX854440 \\
\hline 8 & CSBV-JLCBS-2014 & Changbaishan, Jilin & A. cerana & KU57466 \\
\hline 9 & CSBV-LNDD-2015 & Dandong, Liaoning & A. cerana & KX254334 \\
\hline 10 & CSBV-LNJZ-2015 & Jinzhou, Liaoning & A. cerana & KX254336 \\
\hline 11 & CSBV-LNQY-2015 & Qingyuan, Liaoning & A. cerana & KX254337 \\
\hline 12 & CSBV-GZGY-2015 & Guiyang, Guizhou & A. cerana & KX254332 \\
\hline 13 & CSBV-JXJJ-2015 & Jiujiang, Jiangxi & A. cerana & KX254333 \\
\hline 14 & CSBV-SXYL-2015 & Yulin, Shanxi & A. cerana & KU574662 \\
\hline 15 & CSBV-SXXA-2015 & Xian, Shanxi & A. cerana & KX254338 \\
\hline 16 & CSBV-BJ-2015 & Beijing & A. cerana & KX254340 \\
\hline 17 & CSBV-HB-2016 & Tangshan, Hebei & A. cerana & KY363865 \\
\hline 18 & CSBV-HBQHD-2016 & Qinhuangdao, Hebei & A. cerana & KY363866 \\
\hline 19 & CSBV-HNSX-2016 & Songxian,Henan & A. cerana & KY379055 \\
\hline 20 & CSBV-LNHLD-2016 & Huluodao, Liaoning & A. cerana & KY379056 \\
\hline 21 & CSBV-SXYC-2016 & Yuncheng, Shanxi & A. cerana & KY379058 \\
\hline 22 & AcCSBV-SDLY-2016 & Linyi, Shandong & A. cerana & MF150113 \\
\hline 23 & AmCSBV-SDLY-2016 & Linyi, Shandong & A. mellifera & MG733283.1 \\
\hline 24 & CSBV-HBCD-2016 & Chengde, Hebei & A. cerana & MG979656 \\
\hline 25 & CSBV-LNDD-2017 & Dandong, Liaoning & A. cerana & MG979655 \\
\hline 26 & CSBV-SDLC-2017 & Liaocheng,Shandong & A. cerana & MG979657 \\
\hline 27 & CSBV-HBCD-2017 & Chengde, Hebei & A. cerana & MG979658 \\
\hline 28 & CSBV-JLHD-2017 & Huadian, Jilin & A. cerana & MG979659 \\
\hline 29 & CSBV-JLTH-2017 & Tonghua, Jilin & A. cerana & MG979660 \\
\hline 30 & CSBV-HNNY-2017 & Nanyan, Henan & A. cerana & MG979661 \\
\hline 31 & CSBV-JXJJ-2017 & Jiujiang, Jiangxi & A. cerana & MG979662 \\
\hline
\end{tabular}

2 
Table 2 (on next page)

Table 2. Reference strain and its GenBank number 
Table 2. Reference strain and its GenBank number

\begin{tabular}{|c|c|c|c|c|c|c|}
\hline No. & isolated strain & host & GenBank number & No. & isolated strain & GenBank number \\
\hline 1 & CSBV-FZ-201 & A. cerana & KM495267 & 23 & AmSBV-Kor2-2016 & KP296801 \\
\hline 2 & CSBV-GZ-2009 & A. cerana & AF251124 & 24 & $\begin{array}{c}\text { AcSBV-Kor4-2016 } \\
\text { A. mellifera }\end{array}$ & KP296803 \\
\hline 3 & CSBV-GD-2002 & A. cerana & AF469603 & 25 & AmSBV-Kor1-2016 & KP296800 \\
\hline 4 & CSBV-BJ-2012 & A. cerana & KF960044 & 26 & AcSBV-Indk1A-2013 A. cerana & JX270796 \\
\hline 5 & AcSBV-V1-2014 & A. cerana & KM884990 & 27 & AcSBV-IndII-2-2013 A. cerana & JX270795 \\
\hline 6 & AcSBV-V2-2014 & A. cerana & KM884991 & 28 & AcSBV-IndiaS2-2013 A. cerana & JX270799 \\
\hline 7 & AcSBV-V3-2014 & A. cerana & KM884992 & 29 & AcSBV-K3A-2013 A. cerana & JX270798 \\
\hline 8 & AmSBV-V4-2014 & A. mellifera & KM884993 & 30 & AcSBV-Indiall10-2013 A. cerana & JX194121 \\
\hline 9 & AcSBV-V5-2014 & A. cerana & KM884994 & 31 & AcSBV-Indiall9-2013 A. cerana & JX270800 \\
\hline 10 & AcSBV-VHYnor-20 & 14 A. cerana & KJ959614 & 32 & AcSBV-IndK5B-2013 A. cerana & JX270797 \\
\hline 11 & AmSBV-Viet6-2014 & A. mellifera & KM884995 & 33 & AmSBV-Sweden-2017 A. mellifera & KY273489 \\
\hline 12 & AcSBV-VBP-201 & 7. A. cerana & KX668139 & 34 & AmSBV-NT-2017 A. mellifera & KY465679 \\
\hline 13 & AcSBV-VNam-201 & 15 A. cerana & KJ959613 & 35 & AmSBV-QLD-2017 A. mellifera & KY465678 \\
\hline 14 & AcSBV-VBG-201 & 7 A. cerana & KX668141 & 36 & AmSBV-WA1-2017 A. mellifera & KY465672 \\
\hline 15 & AmSBV-VNA-2017 & A. mellifera & KX668140 & 37 & AmSBV-WA2-2017 A. mellifera & KY465671 \\
\hline 16 & AcSBV-SBM2-201 & 13 A. cerana & KC007374 & 38 & AmSBV-TAS-2017 A. mellifera & KY465676 \\
\hline 17 & CSBV-CQ-2012 & A. cerana & KC285046 & 39 & AmSBV-SA-2017 A. mellifera & KY465677 \\
\hline 18 & CSBV-JXNC-201 & 3 A. cerana & KM232611 & 40 & AmSBV-VN2-2017 A. mellifera & KY465674 \\
\hline 19 & AmSBV-Kor19-2012 & 2 A. mellifera & JQ390592 & 41 & AmSBV-VN1-2017 A. mellifera & KY465675 \\
\hline 20 & AcSBV-Kor-2011 & A. cerana & HQ322114 & 42 & AmSBV-VN3-2017 A. mellifera & KY465673 \\
\hline 21 & CSBV-SXnor-2012 & A. cerana & KJ000692 & 43 & AmSBV-Australia-2014A. mellifera & KJ629183 \\
\hline 22 & AmSBV-Kor21-2012 & 2 A. mellifera & JQ390591 & 44 & AmSBV-UK-2000 A. mellifera & AF092924 \\
\hline
\end{tabular}

2 
Table 3 (on next page)

Table 3. Synthetic oligonucleotides for amplification of the CSBV genome. 
Table 3. Synthetic oligonucleotides for amplification of the CSBV genome.

\begin{tabular}{llc}
\hline Primers & \multicolumn{1}{c}{ Sequence $\left(\right.$ ( $^{\prime}$ to 3 $\left.^{\prime}\right)$} & Nucleotide position $^{\text {s }}$ \\
\hline S1 F & GAAATAAGAATACGAATCGT & $1-20$ \\
S1 R & T AAACAAATCGGTATAAGAGTCC & $379-401$ \\
S2 F & GACCCGTTTTCTTGTGAGTTTTAG & $41-64$ \\
S2 R & GTGTAGCGTCCCCCTGAATAGAT & $611-633$ \\
S3 F & CGTAATTGCGGAGTGGAAAGAT & $273-294$ \\
S3 R & CCCCTAAATTGTTGCGTTGGTT & $739-760$ \\
S4 F & TATTCAGGGGGACGCTACAC & $614-633$ \\
S4R & TATTCCATCGGGGTTATTTG & $1713-1732$ \\
S5 F & GGAGACGCGCATGGTAAAGA & $1644-1663$ \\
S5 R & GCGCGGTAAATAAACACTCG & $2365-2384$ \\
S6 F & ATGGGGGTAAGGGACAATCTG & $2290-2310$ \\
S6 R & TGCTCTAACCTCGCATCAAC & $3423-3442$ \\
S7 F & TTACGGGAGCAGCACAACA & $3391-3409$ \\
S7 R & ATTTCCGATTTACCGATACC & $4287-4306$ \\
S8 F & CGGTGCGTTATGAACCTTTT & $4243-4262$ \\
S8 R & AATGCGTAGATTGAGGTGCC & $5333-5352$ \\
S9 F & TACTATCCGCCCCCTAAGC & $3195-3213$ \\
S9 R & GTGCCCCATCGTTCAAAA & $5432-5449$ \\
S10 F & GCGCAACTGGCACCTCAAT & $5325-5343$ \\
S10 R & TTCCAAATATACTTCCCACTGC & $6249-6270$ \\
S11 F & GCTGGGCCTTCTTATCTGGTG & $3873-3893$ \\
S11 R & TACGGGTCCTCTGCAATGTTCT & $6363-6384$ \\
S12 F & GTGACGGCAGTGGGAAGTAT & $6262-6243$ \\
S12 R & GCAGCCTCCTCAGGTGTTAGT & $7454-7474$ \\
S13 F & TTTGGTAGCGGGGTGTAAG & $7322-7340$ \\
S13 R & CATTGCGTGGTATCATT & $8501-8517$ \\
\hline & & \\
\hline
\end{tabular}


Table 4 (on next page)

Table4.Experimental results of larval challenge 
1

Table 4.Experimental results of larval challenge

\begin{tabular}{cccc}
\hline Group & $\begin{array}{c}\text { The number of dead larvae at } \\
\text { different time points }(\mathrm{a} / \mathrm{b} / \mathrm{c})\end{array}$ & mortality $(\%)$ & $95 \% \mathrm{CI}(\%)$ \\
\hline 1 & $7 / 8 / 9$ & $35-45$ & $19 \sim 64$ \\
2 & $13 / 15 / 14$ & $65-75$ & $46 \sim 88$ \\
3 & $17 / 16 / 18$ & $80-90$ & $62 \sim 97$ \\
4 & $20 / 20 / 20$ & 100 & $83 \sim 100$ \\
5 & $20 / 20 / 20$ & 100 & $83 \sim 100$ \\
6 & $4 / 4 / 5$ & $20-25$ & $6 \sim 44$ \\
\hline
\end{tabular}

2

* a, b and c represent the number of dead larvae from three repeated experiments 
Table 5 (on next page)

Table5. Recombinants detected with RDP 
Table5. Recombinants detected with RDP.

\begin{tabular}{lllll}
\hline Recombinant & Major parent & Minor parent & Breakpoint & Av. P-Val \\
\hline CSBV-JLCBS- & CSBV-FZ-2014 & CSBV-LNQY-2008 & $520-1277$ & $1.048 \times 10^{-18}$ \\
2014 & AmCSBV-SDLY-2016 & CSBV-LNQY-2008 & $2212-3247$ & $8.240 \times 10^{-18}$ \\
& CSBV-FZ-2014 & CSBV-LNQY-2008 & $7202-8336$ & $7.771 \times 10^{-17}$ \\
\hline
\end{tabular}

2 
Figure 1

Phylogenetic analysis of the VP1 gene equencesobtained from all the CSBV isolates and reference strains in GenBank

The tree reveals two clusters, the AC and AM genotypes

* To simplify the naming of the strain, we used AmSBV and AmCSBV to represent the SBVs/CSBV strains isolated from $A$. mellifera; all the others represent the SBVS/CSBV strains that were isolated from $A$. cerana. The corresponding host and gene numbers are listed in Tables 1 and 2 


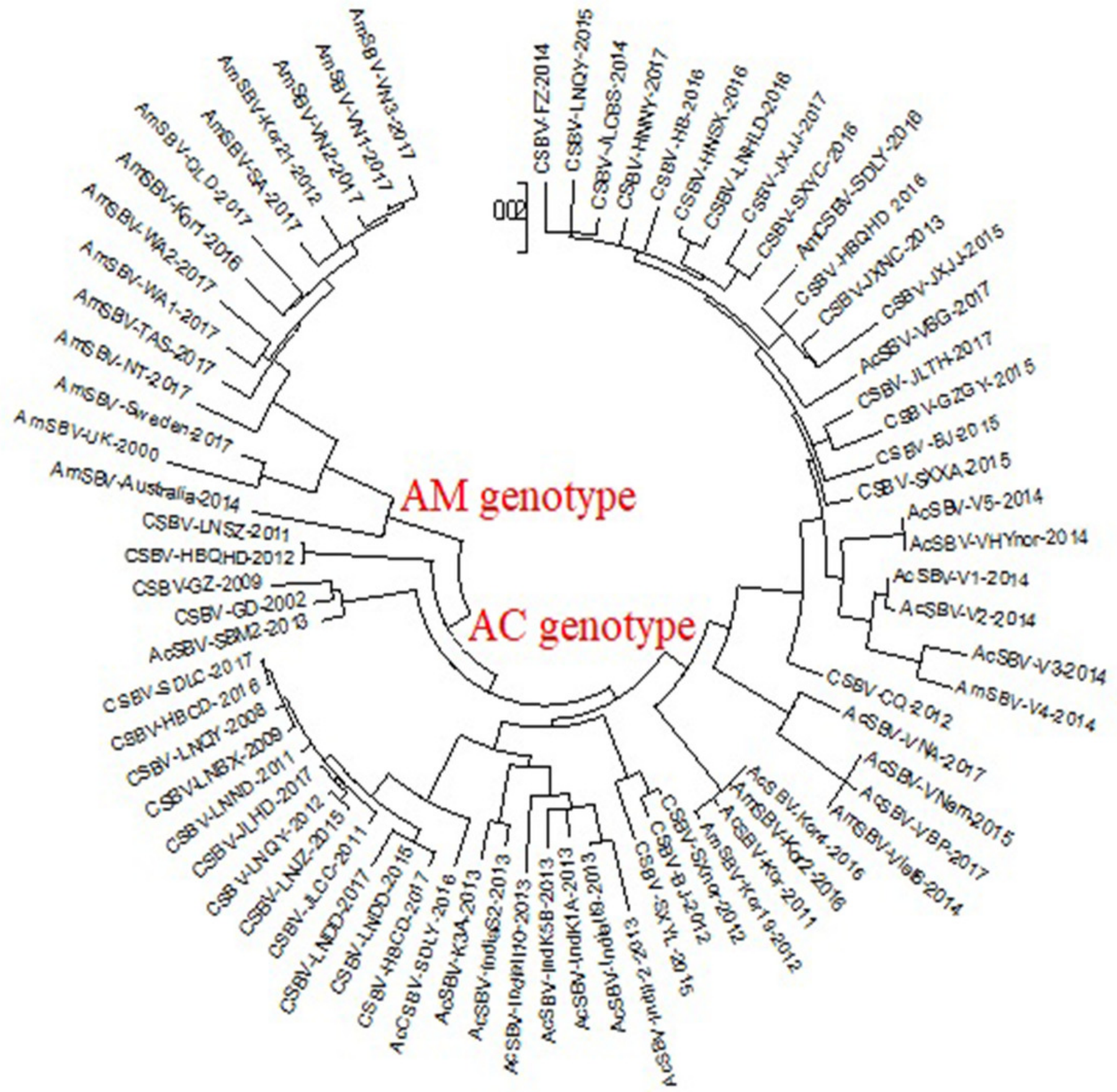


Figure 2

CSBV particles were observed with electron microscopy.

Virus particles of approximately 26-nm diameter were observed in virus preparations from the infected larvae by electron microscopy 


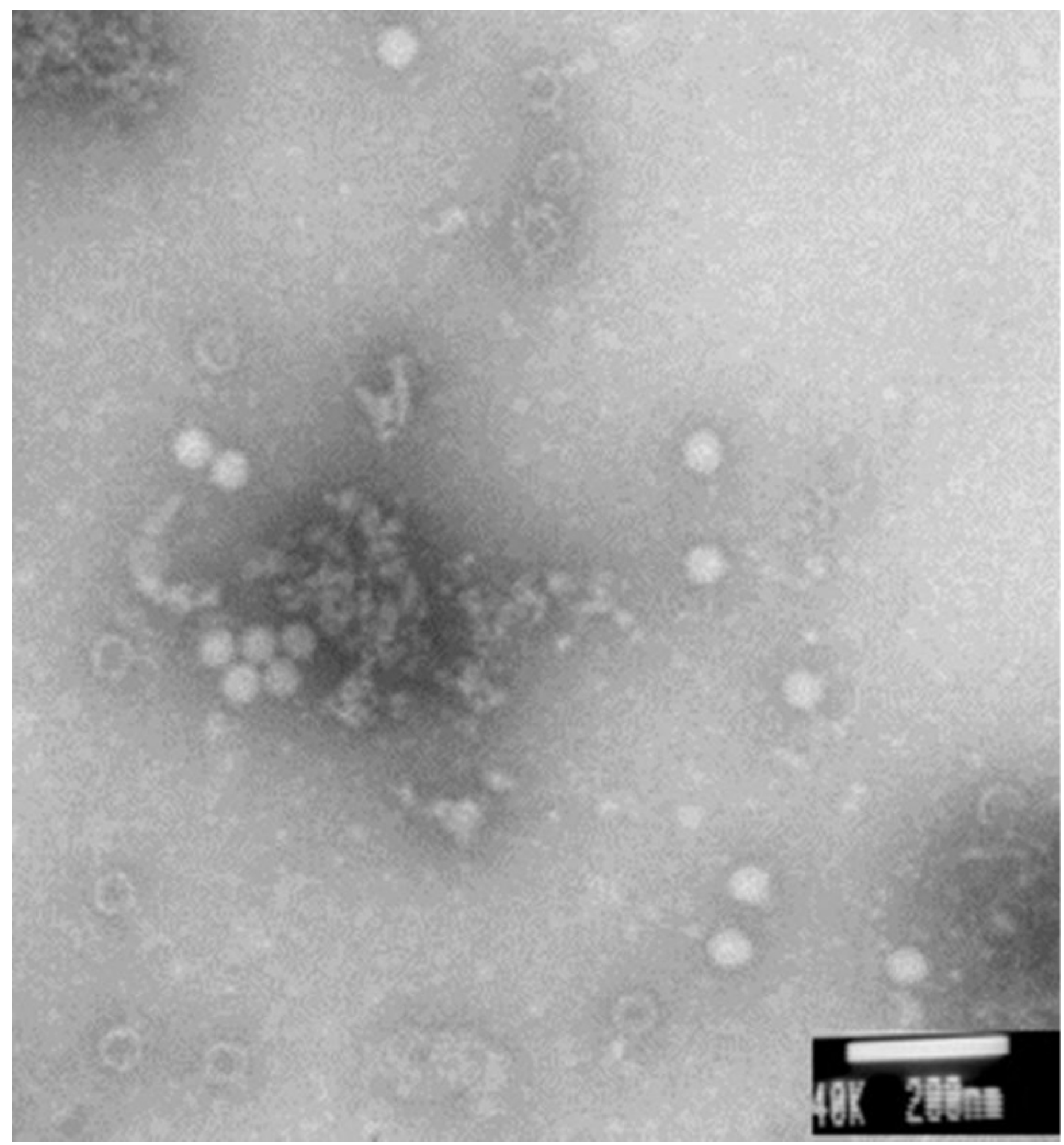


Figure 3

The four proteins of CSBV were detected by SDS-PAGE.

The proteins were resolved on $12 \%$ SDS-polyacrylamide gels according to standard protocols. A. representative protein markers. B. virus-free control. C. AmCSBV-SDLY

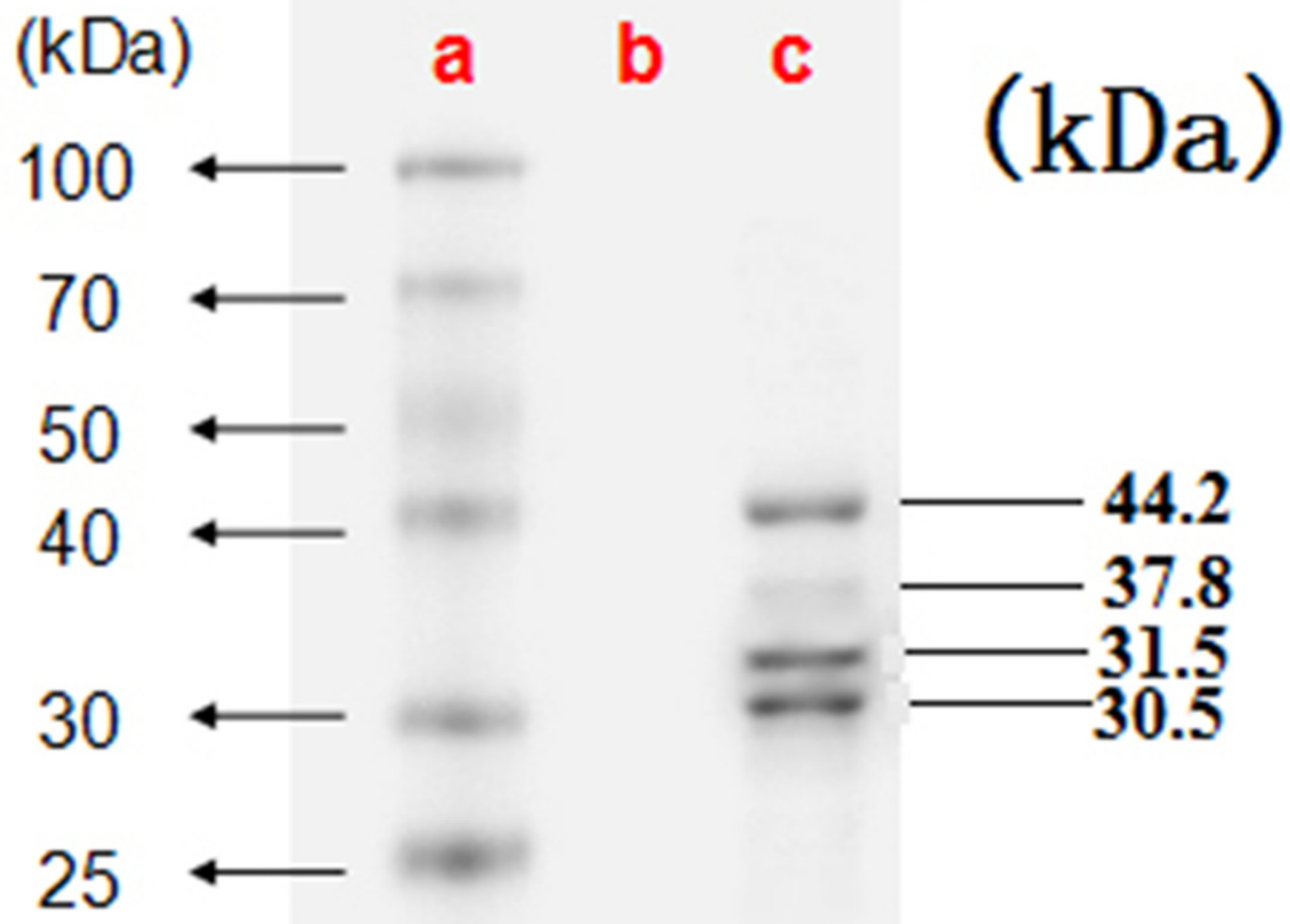


Figure 4

Identification ofAmCSBV-SDLY by AGID assay

Antisera CSBV (center well), AmCSBV-SDLY (wells 1 and 2), known positive control CSBVJL2014-KU574661.1 (wells 3 and 4), and negative control (wells 5 and 6) 


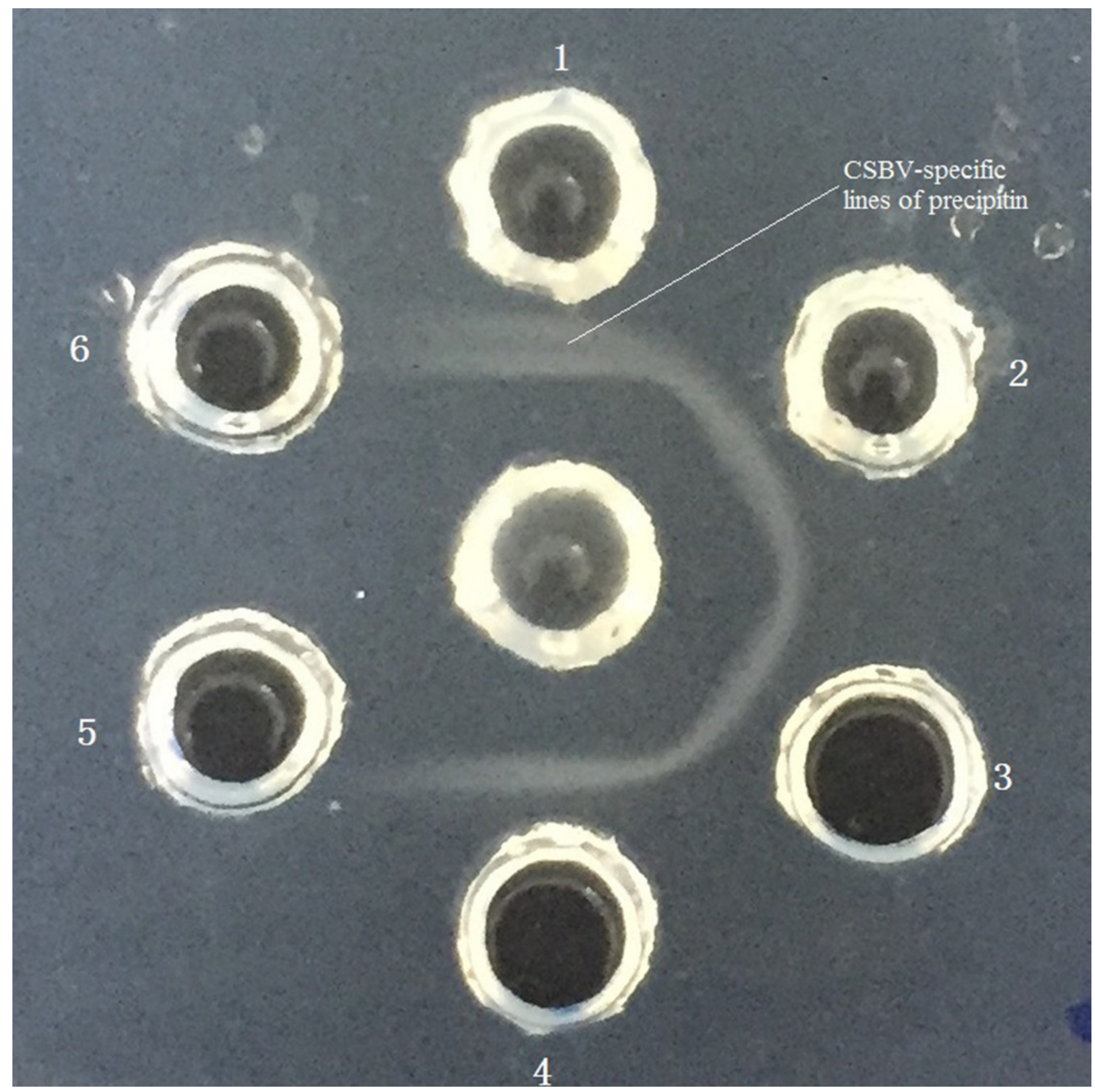


Figure 5

Protein domain alignments

AmCSBV-SDLY-2016 had 17 and 1 amino acid deletions (positions 711-729 and 2,128, respectively). However, AmCSBV-SDLY-2016 had 3 and 13 amino acid deletions (positions 711-713 and 715-728, respectively) as compared with AmSBV-UK-2000 
+ Consensus $\| 1$

Consensus \#1

Majority

AmCSBV-SDLY-2016.pro

AcSBV-Kor-2011.pro AcSBV-Kor 4-2016.pro AcSBV-V1-2014.pro AcSBV-V2-2014.pro AcSBV-V3-2014.pro AcSBV-V5-2014.pro AcSBV-VHYnor-2014.pro AmSBV-V4-2014.pro AmSBV-Kor19-2012.pro CSBV-JLCBS-2014.pro AcSBV-VNA-2017.pro CSBV-FZ-2014.pro CSBV-LNQY-2009.pro CSBV-GD-2002.pro CSBV-BJ-2012.pro CSBV-SXnor-2012.pro CSBV-SXYL-2015.pro AmSBV-Viet6-2014.pro AcSBV-VNam-2015.pro AcSBV-VBP.2017.pro AmSBV-Kor2-2016.pro AmSBV-UK-2000.pro

+ Consensus $\# 1$

Consensus \#1 Majority

AmCSBV-SDLY-2016.pro AcSBV-Kor-2011.pro AcSBV-Kor 4-2016.pro AcSBV-V1-2014.pro AcSBV-V2-2014.pro AcSBV-V3-2014.pro AcSBV-V5-2014.pro AcSBV-VHYnor-2014.pro AmSBV-V4-2014.pro AmSBV-Kor19-2012.pro CSBV-JLCBS-2014.pro AcSBV-VNA-2017.pro CSBV-FZ-2014.pro CSBV-LNOY-2009.pro CSBV-GD-2002.pro CSBV-BJ-2012.pro CSBV-SXnor-2012.pro CSBV-SXYL-2015.pro AmSBV-Viet6-2014.pro AcSBV-VNam-2015.pro AcSBV-VBP-2017.pro AmSBV-Kor2-2016.pro AmSBV-UK-2000.pro

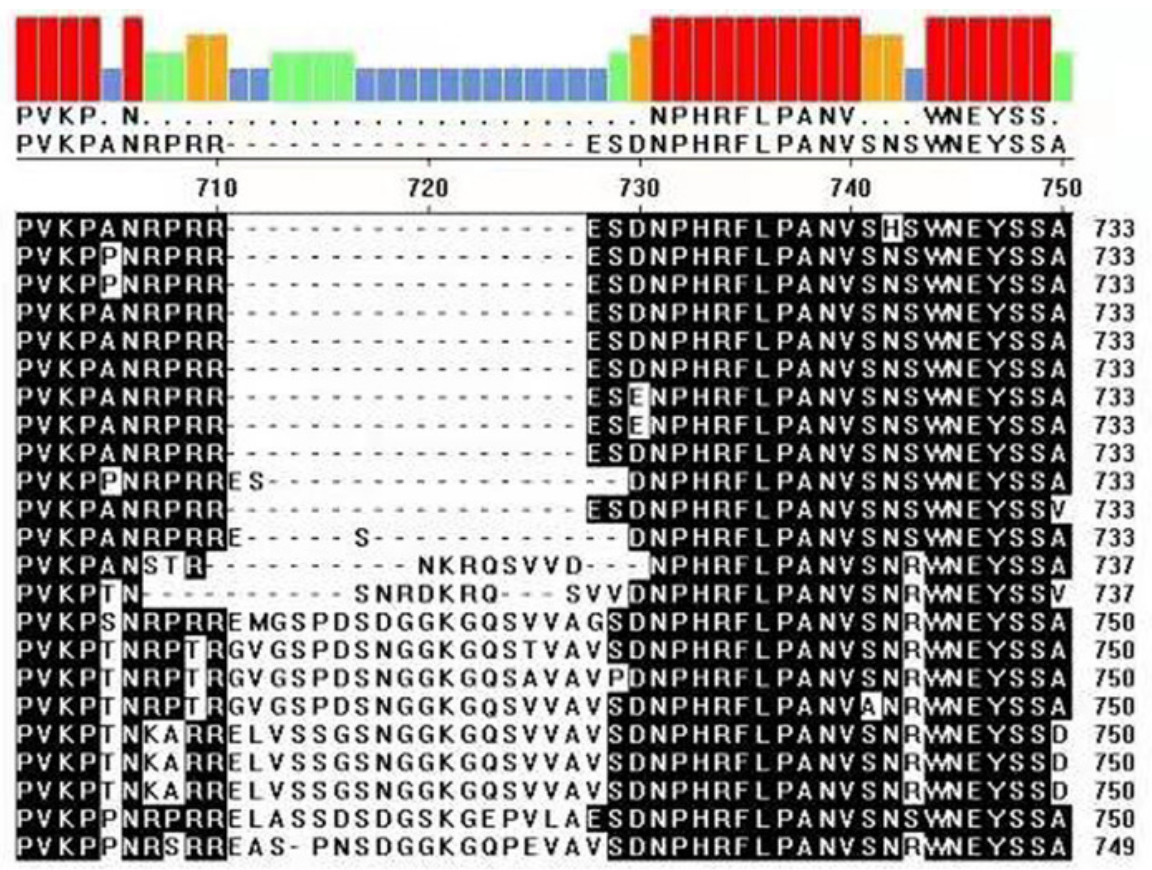

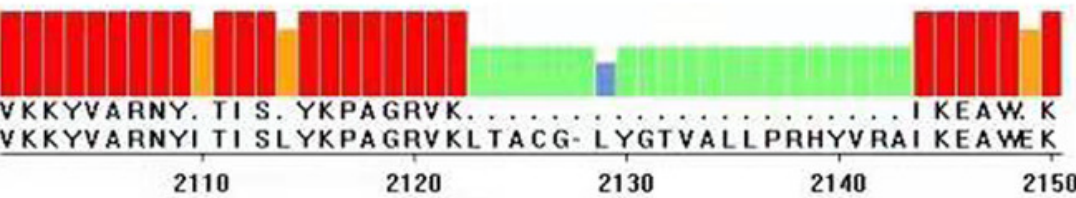

VKKYVARNYI TI SLYKPAGRVKLTACG LYGTVALLPRHYVRAI KEAWEK 2132 VKKYVARNYI TI SLYKPAGRVKLTACG- I YGTVALLPRHYVRAI KEAWEK 2132 VKKYVARNYI TI SLYKPAGRVKLTACG, I YGTVALLPRHYVRAI KEAWEK 2132 $\checkmark K K Y V A R N Y I$ T I SLYKPAGRVKLTACG- LYGTVALLPRHYVRAI KEAWEK 2132 VKKYVARNYI TI SLYKPAGRVKLTACG- LYGTVALLPRHYVRAI KEAWEK 2132 VKKYVARNYI TI SLYKPAGRVKLTACG LYGTVALLPRHYVRAI KEAWEK 2132 VKKYVARNYI TI SLYKPAGRVKLTACG LYGTVALLPRHYVRAI KEAWEK 2132 $\checkmark K K Y V A R N Y I$ TI SLYKPAGRVKLTACG IYGTVALLPRHYVRAI KEAWEK 2132 $\checkmark K K Y V A R N Y I$ TI SLYKPAGRVKLTACG LYGTVALLPRHYVRAI KEAWEK 2132 VKKYVARNYI TI SLYKPAGRVKLTACG IYGTVALLPRHYVRAI KEAWEK 2132 $\checkmark K K Y V A R N Y I$ TI SLYKPAGRVKLTACG LYGTVALLPRHYVRAI KEAWEK 2132 VKKYVARNYI TI SLYKPAGRVKLTACG IYGTVALLPRHYVRAI KEAWEK 2132 VKKYVARNYI TI SLYKPAGRVKVNCMWPLWDSGSI TEALCAVPI KEAWEK 2137 $\checkmark K K Y V A R N Y V T$ I SLYKPAGRV KVNCMRHL WDSGSI TEALCAVPI KEAWDK 2137 VKKYVARNYI TI SLYKPAGRV KVNCMWHL WDSGLVT QAFCAVPI KEAWEK 2150 VKKYVARNYI TI SLYKPAGRV KVNRMWYL WDSGFI TEAL CAVPI KEAWEK 2150 VKKYVARNYI TI SLYKPAGRVKVNRMWYL MDSGFI TEALCAVPI KEAWEK 2150 VKKYVARNYI TI SLYKPAGRVKLTACG I YGTVALLPRHYVRAI KEAWEK 2149 IKKYVARNYI TI SLYKPAGRVKLTACG I YGTVALLPRHYVRAI KEAWEK 2149 VKKYVARNYI TI SLYKPAGRVKLTACG I YGTVALLPRHYVRAI KEAWEK 2149 VKKYVARNYI TI SMYKPAGRVKLTACG I YGTVALLPRHYVRAI KEAWEK 2149 VKKYVARNYI TI SLYKPAGRVKLTACG I YGTVALLPRHYVRAI KEAWEK 2149 VKKYVARNYI TI SLYKPAGRVKLTACG I YGTVALLPRHYVRAIKEAWEK 2148 


\section{Figure 6}

Phylogenetic analysis of all nucleotide sequencesobtained from various countries, including China, Korea, Vietnam, India,Australia, and the United Kingdom.

Numbers on the nodes indicate clade credibility values. Previously reported sequence names are presented in the following format: strains-GenBank accession numbers. The tree reveals two clusters, the AC and AM genotypes.

* The bar represents a genetic distance of 0.01 . CSBV is marked with a red box, the blue square represents that the AC genotype SBVs strains mainly were isolated from A. mellifera.

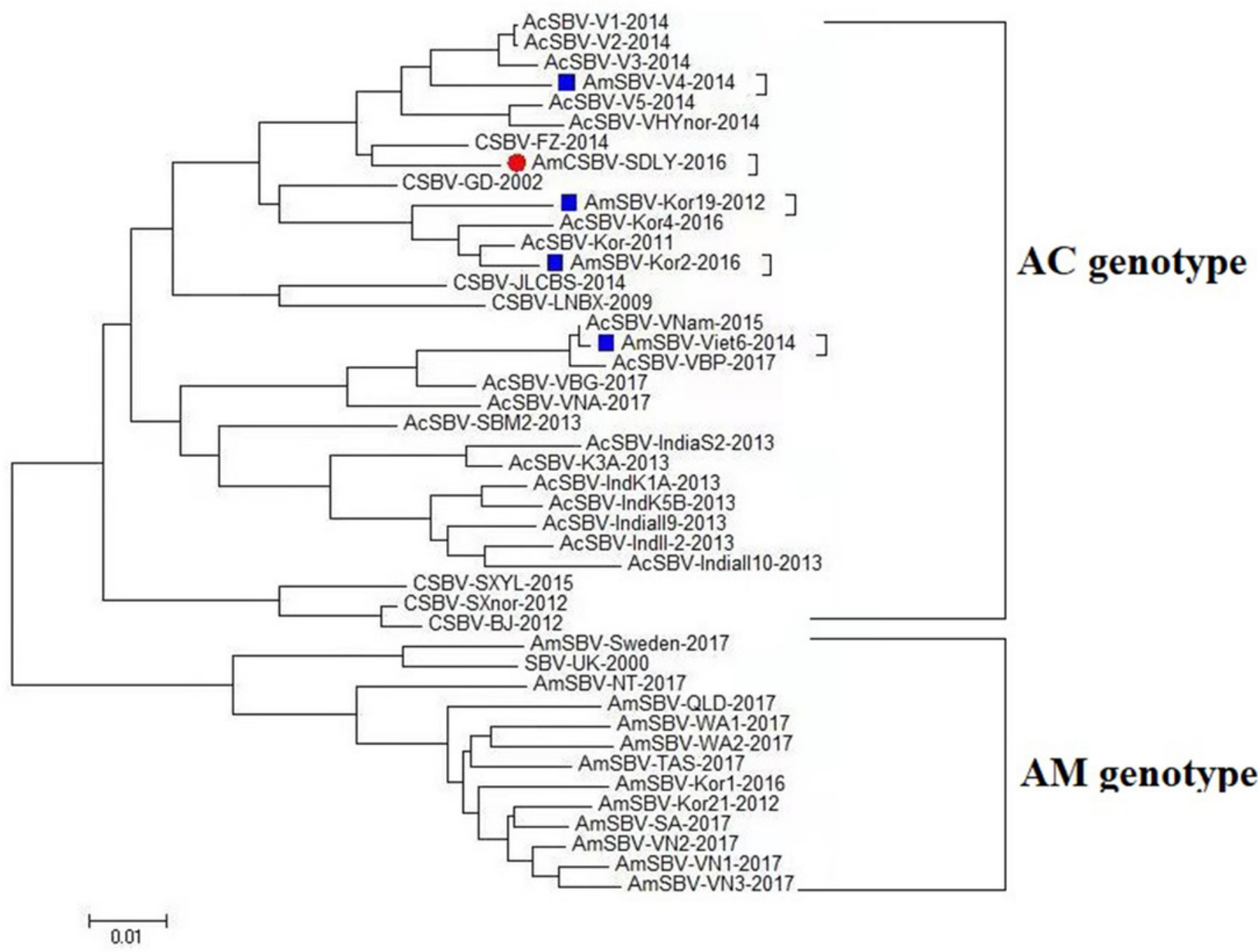


Figure 7

SimPlot graphscomparing AmCSBV-SDLY-2016 with other CSBVs and AmSBV-UK-2000

Each colored line indicates a group of SBV/CSBV strains (for interpreting the color key in this figure legend, please refer to an online version of the article)

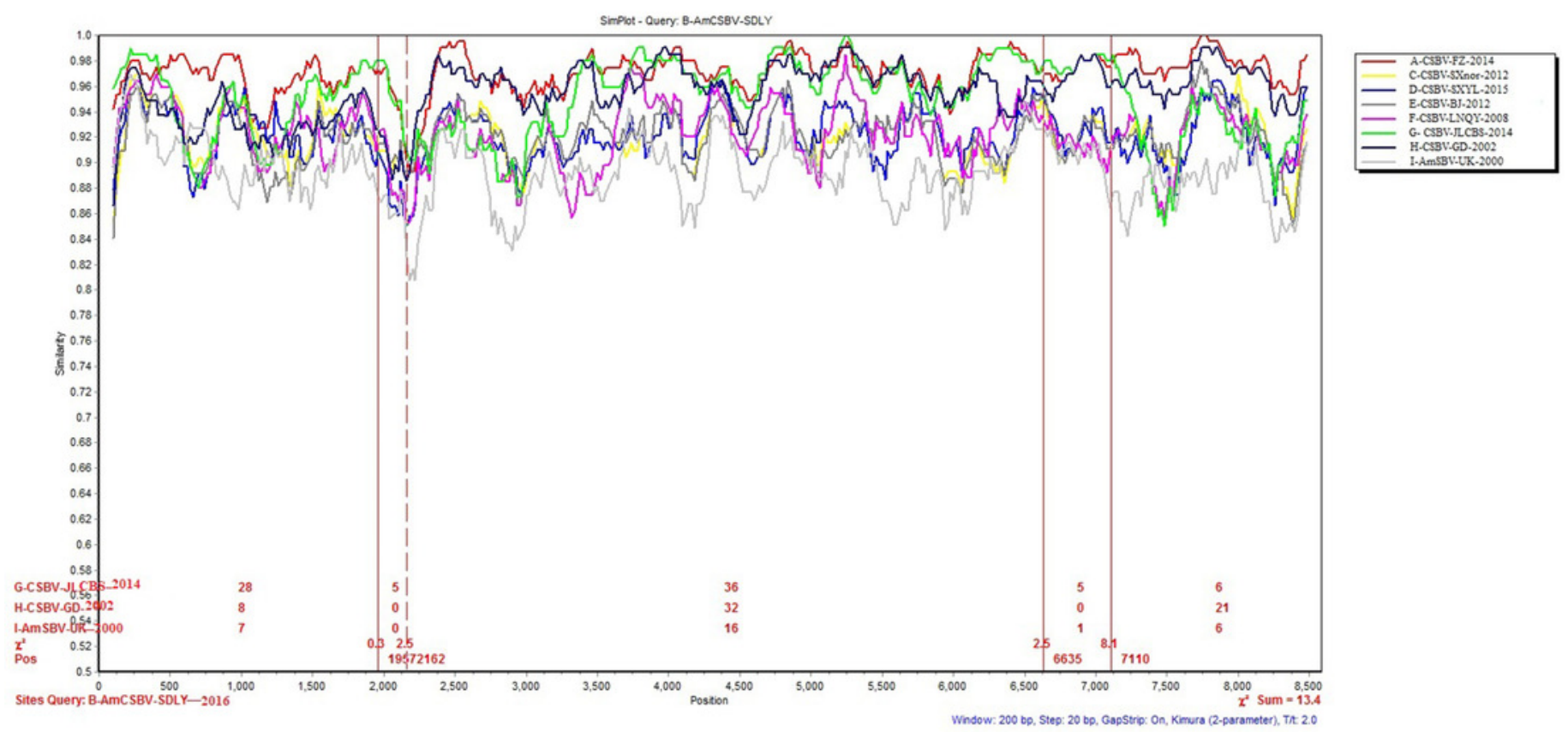


Figure 8

BootScan analysis of the recombinant sequence based on the comparison between the complete coding regions of AmCSBV-SDLY-2016 and other CSBVs and AmSBV-UK-2000

A. BootScan was conducted with the strain AmCSBV-SDLY-2016 as the query sequence. B. BootScan was conducted with the strain CSBV-JLCBS-2014 as the query sequence. Note: The cross recombination position is labeled by red lines. 

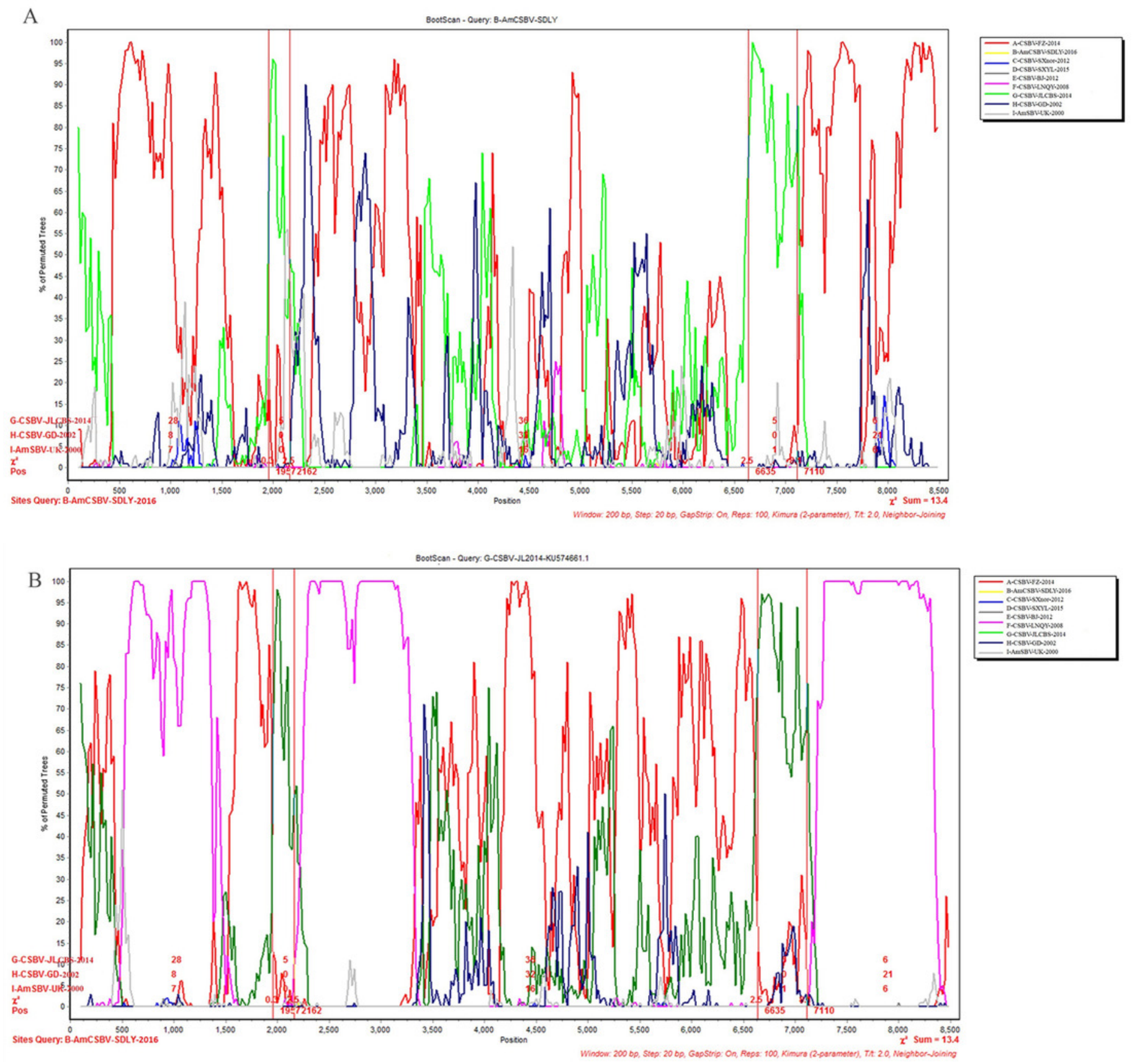